reduce RV cavity size in rTOF may be beneficial. As such, obesity is a promising and modifiable therapeutic target in rTOF.

Conflict of Interest None

\section{CRYPTOGENIC STROKE AND PATENT FORAMEN OVALE DEVICE CLOSURE: THE IMPORTANCE OF MULTI- DISCIPLINARY DECISION-MAKING}

${ }^{1}$ Sashiananthan Ganesananthan*, ${ }^{1}$ Carven Yee Shean Chin, ${ }^{1}$ Katie W.L. Kwan, ${ }^{1}$ Timothy Woo, ${ }^{1}$ Zong Xuan Lee, ${ }^{2}$ Tom Hughes, ${ }^{2}$ Peter Groves. ${ }^{1}$ Cardiff University School Of Medicine; ${ }^{2}$ University Hospital UK

\subsection{6/heartjnl-2019-BCS.138}

Introduction There is a high prevalence of patent foramen ovale (PFO) in patients with cryptogenic stroke; paradoxical embolism may be implicated in some but not all of these. Percutaneous device PFO closure reduces the risk of recurrent emboli but the success of this treatment is dependent on appropriate patient selection. The work-up of patients with cryptogenic stroke and PFO is best undertaken by a multi-disciplinary team (MDT).

Aim The aim was to evaluate the impact of a methodical approach to MDT investigation and work-up of patients with cryptogenic stroke and PFO on the final diagnosis and selection of patients for device PFO closure in a UK centre.

Methods The study was done in a single tertiary cardiac centre providing a PFO device closure service to a population of 1.3 million. Consecutive patients referred to the service between March 2011 and January 2017 were studied. Assessment included clinical consultation and expert review of all cardiac imaging (TOE and bubble contrast TTE), brain imaging (CT, DWI MRI, CT angiography), thrombophilia and arrhythmia investigations. Information about the MDT conclusions was obtained from meeting minutes, electronic and paper patient records.

Results 171 patients [51\% female, mean age of 42 years (SD: \pm 16$)]$ were assessed. The median number of MDT discussions was 1 per patient (range 1-5). Referral was with a cerebral infarct in $82 \%$, transient cerebral ischaemia in $12 \%$ and peripheral emboli in $6 \%$. Brain imaging confirmed the presence of cerebral infarction in $74 \%$ of patients. Cardiac imaging confirmed the presence of a PFO in $88 \%$, an ASD in $5 \%$ and both in $4 \%$; the intra-atrial septum was intact in $3 \%$. One 'high risk' echo marker for paradoxical embolism was present in $44 \%$ of patients; an atrial septal aneurysm (23\%), large right-to-left shunt $(20 \%)$ or spontaneous shunt (43\%). Clinically significant atrial arrhythmia was detected in $5 \%$ and thrombophilia testing was abnormal in 5\% with lupus anticoagulant being positive in $62 \%$ of this subgroup. The final diagnoses are summarized in the figure 1. Paradoxical embolism was proposed if there was cerebral infarction typical of thrombo-embolism in the absence of vascular disease or arrhythmia and in the presence of PFO or ASD with a 'high-risk' ECHO marker. Based on these criteria, device closure was recommended in $41 \%$ of patients. The remainder received treatment appropriate to their diagnosis: antiplatelet therapy in 36\%, anti-coagulants in $10 \%$ and no treatment in $15 \%$.

Conclusion In patients with cryptogenic stroke who have a PFO, paradoxical embolism is implicated in the minority. Methodical work-up of patients by an MDT results in a range of diagnoses most of which are unrelated to the PFO. While clinical follow-up is required in all patients included
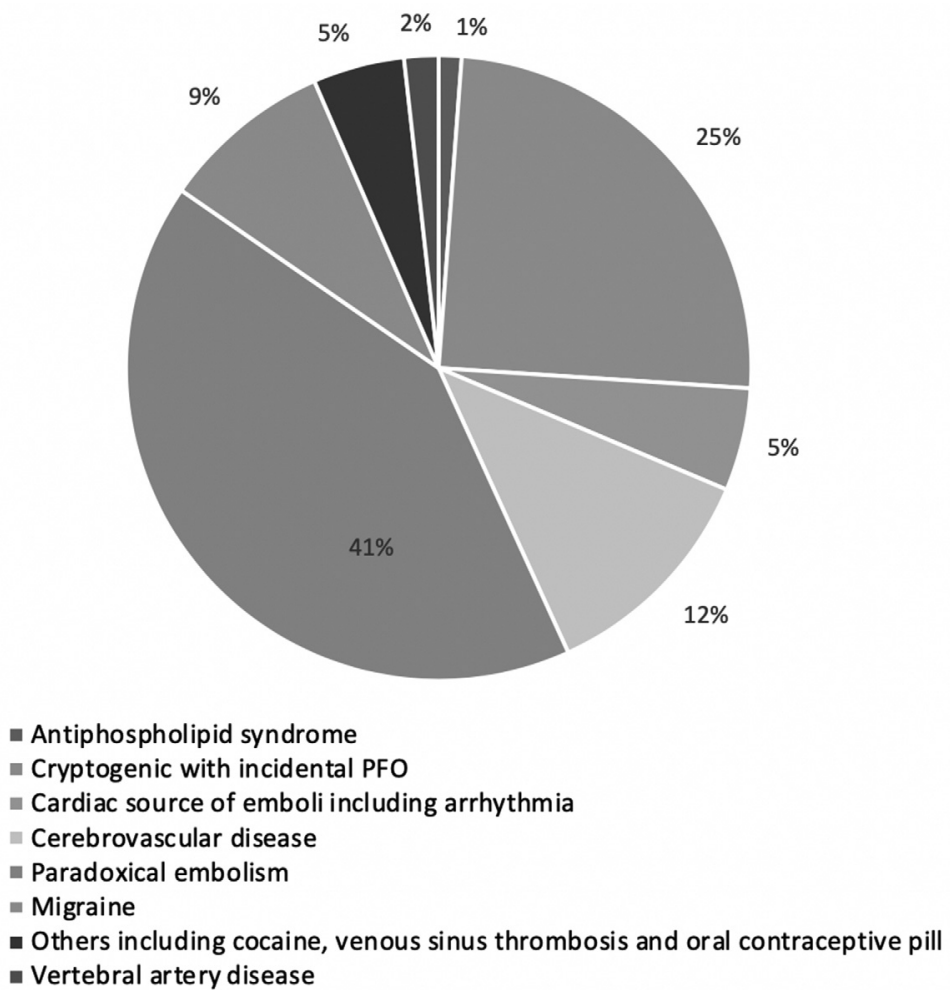